\title{
Comparative early results of a robotics-assisted endoscope holder in single port thoracoscopic surgery in the era of COVID-19
}

\author{
Ching Feng $\mathrm{Wu}^{1,2} \cdot$ Ching Yang Wu ${ }^{1} \cdot$ Yin Kai Chao ${ }^{1} \cdot$ Diego Gonzalez-Rivas $^{2} \cdot$ Ming Ju Hsieh $^{1}$ (D $\cdot$ Yu Bin Pan $^{3}$. \\ Lan Yan Yang ${ }^{3}$
}

Received: 29 July 2021 / Accepted: 11 January 2022 / Published online: 7 February 2022

(c) The Author(s), under exclusive licence to Springer Science+Business Media, LLC, part of Springer Nature 2022, corrected publication 2022

\begin{abstract}
Background Innovations in surgical instruments have made single-port surgery more widely accepted and lead to a reduced demand for surgical assistants. As COVID-19 has ravaged the world, maintaining minimum medical staffing requirements and proper social distancing have become major topics of interest. We sought to evaluate the feasibility of applying the unisurgeon approach in single-port video-assisted thoracoscopic surgery aided by a robotic camera holder.

Methods Operative time, blood loss, setup time, postoperative hospital stays, and the number of participating surgeons in single-port video-assisted thoracoscopic lung resections were gathered for investigation after the introduction of the ENDOFIX $^{\text {exo }}$ robotic endoscope holder system. In this cohort, we collected 213 patients who underwent single port video thoracoscope surgery, including 57 patients underwent robotic endoscope arm assisted surgery and case-matched 52 patients in the robotic arm-assisted group with patients in the human-assisted group through propensity score-matched analysis.

Results In wedge resection, a single surgeon was able to completely operate on all lobes of target lesions. However, for anatomical resections, namely segmentectomy, the success rate was $95 \%$, and for lobectomy, the success rate was only $64 \%$. No significant differences between setup times, blood loss, or operative times between the two groups were observed.

Conclusions When an experienced uniport surgeon is assisted by a robotic endoscope holder, wedge resection is the most suitable procedure to be performed through unisurgeon single-port video-assisted thoracoscopic surgery without increasing setup time, operative time, or short-term complications. Verification of the technique's applicability for use in anatomic resections requires further investigation.
\end{abstract}

Keywords Unisurgeon · Single-port VATS · Robotic camera holder $\cdot$ Social distancing $\cdot$ COVID-19

\section{Abbreviations}

COVID-19 Coronavirus disease 2019

VATS Video-assisted thoracoscopic surgery

SPVATS Single-port video-assisted thoracoscopic surgery

IRB No: 202002019B0.

Ming Ju Hsieh

hsiehmj2@gmail.com

1 Chang Gung University, Division of Thoracic and Cardiovascular Surgery, Department of Surgery, ChangGung Memorial Hospital, Linkou, Taiwan

2 Coruña University Hospital; Minimally Invasive Thoracic Surgery Unit (UCTMI), Department of Thoracic Surgery, CORUÑA, Spain

3 Biostatistics Unit, Clinical Trial Center, Chang Gung Memorial Hospital, Taoyüan, Taiwan
ASIS Anterior superior iliac spine

HA group Human-assisted group

EA group ENDOFIX ${ }^{\text {exo }}$-assisted group

BMI Body mass index

PACS Picture archiving and communication system

ECOG Eastern Cooperative Oncology Group score

After nearly 30 years of evolution, video assisted thoracoscope surgery (VATS) has proven its safety and feasibility in treating lung diseases [1-5]. In addition to reducing the size and number of wounds, the introduction of robotic in minimal invasive surgery has also become a trend. Okada et al. first proposed and implemented the idea in lung resection [6]. Kunisaki et al. also successfully used the AESOP robotic system (Intuitive Surgical, Sunnyvale, CA, USA) to complete single-surgeon esophagectomy [7]. Thirteen years later, the first unisurgeon single port video assisted 
thoracoscope lobectomy (SPVATS) was completed [8]. However, related reports have been sporadic, and the clinical efficacy of unisurgeon single-port VATS has not been sufficiently evaluated. In the urgent era of the COVID-19 pandemic, appropriate social distancing and surgical staffing are crucial issues to address. Hence, our aim was to optimize the use of existing human resources and maintain appropriate social distance during operations as much as possible. Therefore, we incorporated the use of a robotic endoscope holder into our elective single-port VATS surgery to investigate whether reducing staffing demands and maintaining proper social distancing could be achieved through the use of an assistive robotic endoscope holder.

\section{Materials and methods}

\section{Robotic endoscope holder platform}

ENDOFIX $^{\text {exo }}$ (AKTORmed, Barbing, Germany) is a robotic endoscope holder with computer-controlled electric motors. It has six computer-controlled joints that can be adjusted manually, and the terminal two joints serve as a bionic human wrist. After the scope is docked at the robotic arm, the operator can arbitrarily adjust the endoscope to any angle. Due to its quick-coupling device, it can be easily installed on any part of the side rail of the operation table. After the patient has been placed in the lateral decubitus position, the robotic endoscope holder is usually attached to the operation table rail on the side opposite to the operator and the scope is held to point from anterior to posterior. The robotic endoscope holder is docked above or below the imaginary line running through the anterior superior iliac spine (ASIS). The operation room staff, and robotic arm configurations are depicted in detail in Fig. 1A. The position is adjusted to above or below the ASIS, depending on the target lesion location (Fig. 1B). For example, the robotic arm would be fixed below the ASIS line for upper lobe lung tumor resection. For lower lobe lung tumor resection, the robotic arm would be placed above the ASIS line. A $3-\mathrm{cm}$ wound is created at the pivot of the anterior axillary line and the fourth or fifth intercostal space. A plastic wound protector is used to maintain clear vision of the surgical field when the endoscope enters the thoracic cavity. The 10-mm $30^{\circ}$ video telescope was placed at the posterior end of a wound. After the endoscope had been adjusted according to the target lesion, defining the axis of motion, the surgeon was able to control scope movement in a straightforward and intuitive manner through an ergonomic control key on the upper side of the endoscope fixation site (Fig. 2A). In most cases, no change to the customary working approach is required; To ensure the safety of the operation, all operations were performed by the same surgeon (C.F.W), who had accumulated a wealth of experience in SPVATS and participated in robotic endoscope holder-assisted single-port VATS at Coruña University Hospital. Regarding the robotic endoscope holder configuration for surgical assistance, the following operation staff setup was used: one surgeon, one assistant, one scrub nurse, and one circulating nurse. The footprint stickers on the ground were used to maintain social distancing of $>1 \mathrm{~m}$ (Fig. 2B). The assistant participated in the surgery when the surgeon needed their help, such as retracting the parenchyma to dissect the hilar structure, or when the robotic endoscope holder did not provide adequate surgical vision. The assistant recorded the reason and frequency of provided help, surgical images using foot pedals, and other data to evaluate whether the three types of lung resection could be completed by one surgeon without any help from the assistant.

\section{Patients and selection criteria}

This study was approved by the Chang Gung Memorial Hospital Institutional Review Board (IRB No:
Fig. 1 A Configurations of operation room staff and robotic arm. B The docking landmark was below/above the anterior superior iliac spine (ASIS) according to the lesion location
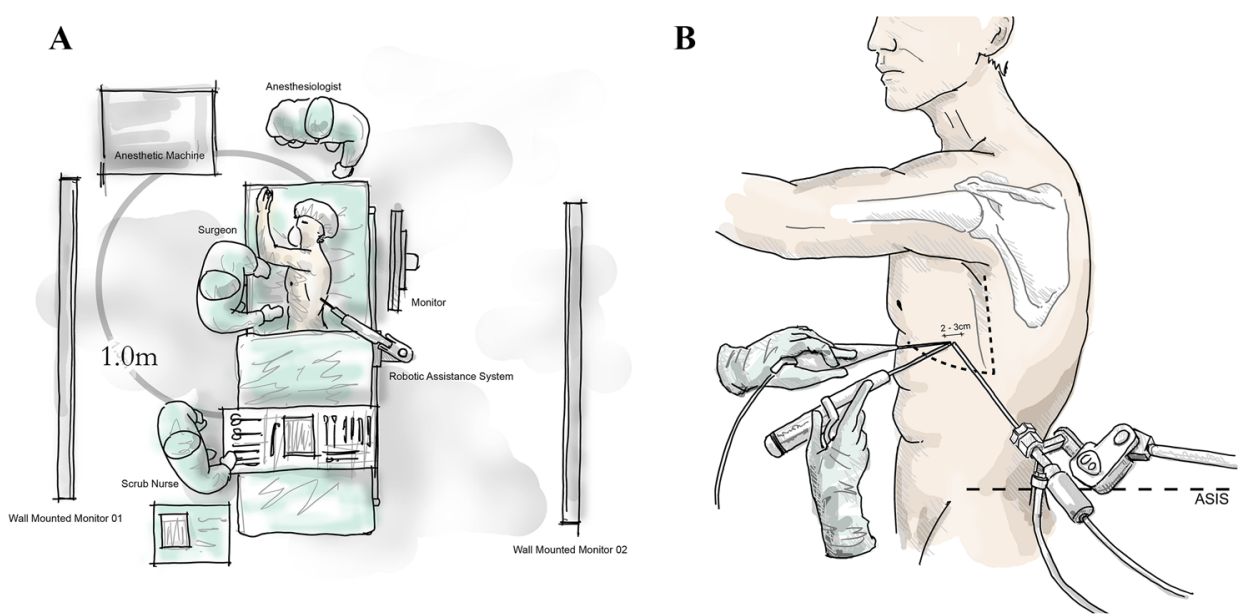
Fig. 2 A The robotic camara holder was controlled by an ergonomic control button, $\mathbf{B}$ Footprint sticker on the ground that was used to maintain social distancing

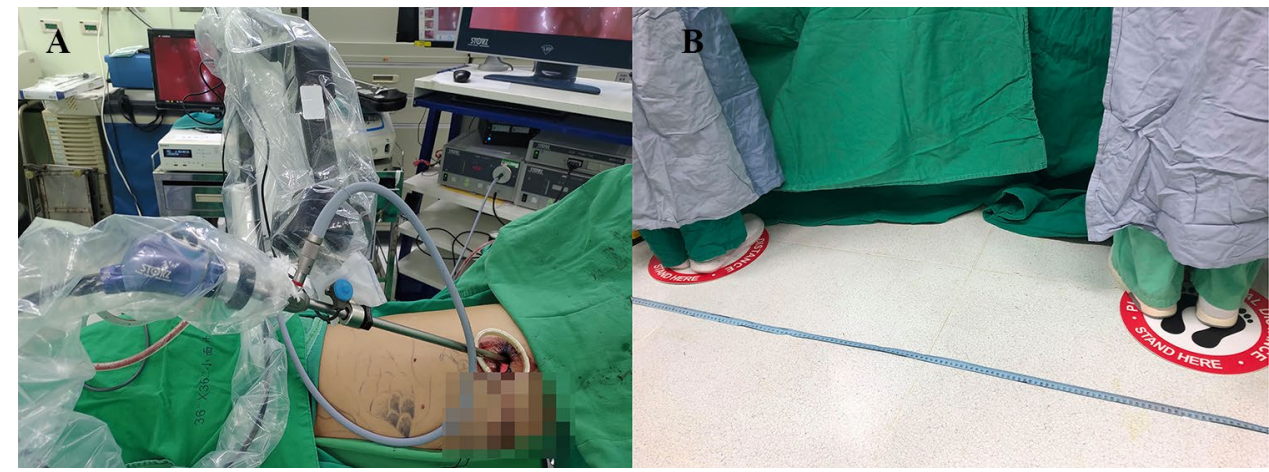

202002019B0). We retrospectively reviewed the medical charts of patients who received VATS pulmonary resection from January 2018 to October 2020. The inclusion criteria for robotic endoscope holder-assisted singleport VATS were patients who (a) Age $>18$ years (b) lung lesion $<5 \mathrm{~cm}$ (c) exhibited normal coagulation function. Surgical procedures were determined in accordance with the need for treatment, whether patients have been identified preoperatively as having primary lung cancer; lobectomy was performed in patients with tumors $>2 \mathrm{~cm}$. Segmentectomy was performed to treat ground glass opacity lesions or tumors $<2 \mathrm{~cm}$. Wedge resection was performed with intent of tissue proof or extrapulmonary malignancy with lung metastasis. Postoperative complications were all collected and classified using a scale from I to V according to the Clavien-Dindo classification. The criterion for drainage tube removal was a drainage amount $<250 \mathrm{~mL} /$ day without air leak.

To objectively evaluate whether the setup time of the robotic endoscope holder affects the result, the time from room entry to wound closure was divided into four stages: time of anesthesia induction, time of operation preparation, operative time, and time of sign out, which were all recorded in our operation record. Surgeries in which a human assistant operated the thoracoscope before the introduction of the robotic endoscope holder were included in the humanassisted (HA) group, and surgeries that were conducted using the robotic endoscope holder were included in the ENDOFIX $^{\text {exo }}$-assisted (EA) group.

To determine whether a difference existed in image quality between the EA and HA groups, we reviewed the surgical images of the two groups after matching. In our surgical routine, images recording are necessary and used for medical insurance claims. The image capture settings were fixed in our PACS (picture archiving and communication system). Surgical images were divided into three zones by two ellipses that were $50 \%$ and $80 \%$ of the surgical field in length and width, and the zones were defined as the central, intermediate, and marginal zones when the target lesion and endostaple were located inside the 50\% ellipse, between the 50 and $80 \%$ ellipses, and outside of the $80 \%$ ellipse, respectively (Fig. 3).

\section{Statistical analyses}

Because different backgrounds of the enrolled patients and variations in surgeries could affect the outcome, propensity matching was conducted. For propensity score calculation, we conducted a logistic regression of the following factors: age, body mass index (BMI), Eastern Cooperative Oncology Group score (ECOG), diagnosis (benign/malignant), lesion location, and operative method (wedge/anatomic resection); propensity matching at the $1: 1$ ratio was performed using the nearest neighbor matching method. Continuous data were analyzed using Student's $t$ test or the Mann-Whitney $U$ test. Fisher's exact test or chi-squared test was used for comparison of categorical data. SPSS version 25 was used for statistical analyses. All tests were two-sided, and $P<0.05$ was considered statistically significant.

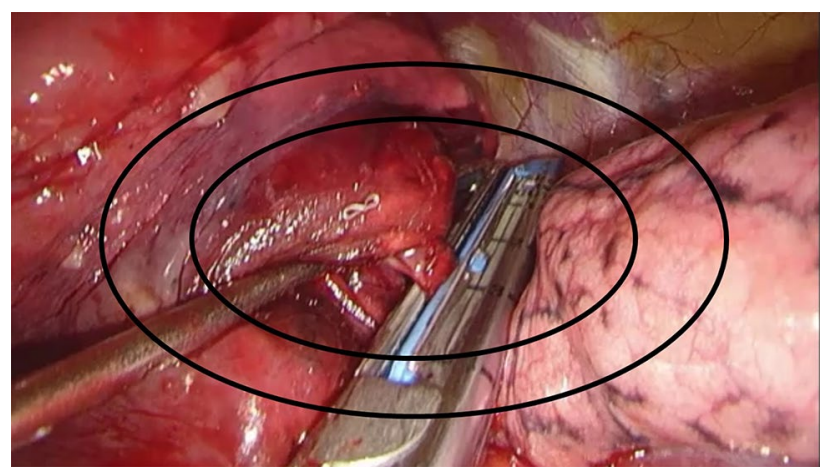

Fig. 3 Image quality evaluation. The operative field was divided into three areas by two ellipses $50 \%$ and $80 \%$ of the length and width of surgical image 


\section{Results}

A total of 2676 patients from our institution underwent VATS surgery between January 2018 and October 2020. A total of 213 patients received elective single port VATS surgery, including 156 lung resections performed by human assisted single port VATS surgery between January 2018 and March 2020 and 57 lung resections intentionally performed by using the robotic endoscope holder between April 2020 and October 2020 (comprising 23 wedge resections and 34 anatomic resections). These surgeries were matched at a 1:1 ratio with controls in a retrospective history comparative study. A total of 52 paired surgeries were included in the final unisurgeon feasibility and intraoperative image quality analysis (Fig. 4). The patients' characteristics and perioperative results before and after propensity matching are shown in Table 1. Before matching, only ECOG showed significant difference $(P=0.002)$. After matching, there are no obvious difference between two groups. As for perioperative results, there are no significant difference observed in the preoperative preparation time, intraoperative blood loss, drainage tube duration, postoperative hospital stays, or other factors. Complete perioperative results are listed in Table 2 and supplementary Table 1. Significant differences were found in only the surgeon demand during surgery. In terms of the different surgical methods, wedge resection could be completed by one surgeon, and the assistant's help was required in one case of segmentectomy (1/20) and in five cases of lobectomy (5/14). Even after propensity score matching, no difference was found in operation preparation, operative time, or surgery-related complications. Significant differences were still discovered in only the number of surgeon involved in the surgery $(P<0.01)$. In terms of the feasibility of unisurgeon SPVATS, wedge resection could be performed

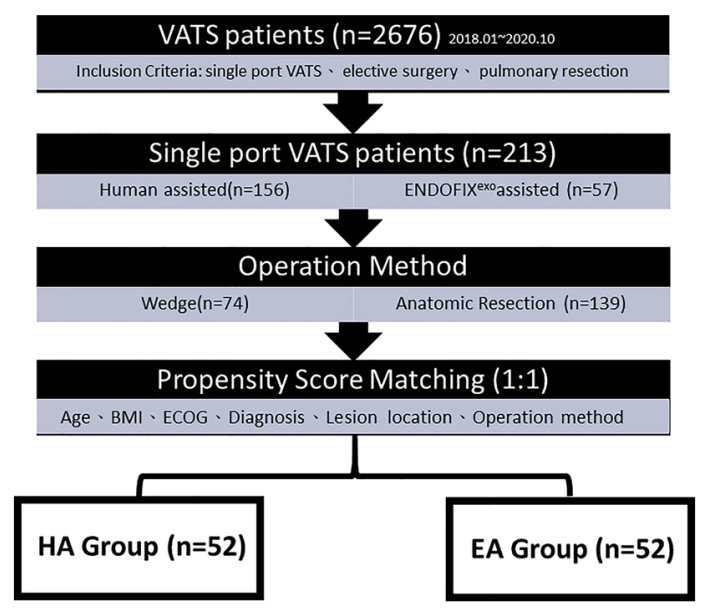

Fig. 4 Flow diagram of the progress through the study by one surgeon. In terms of anatomic resection, the feasibility of segmentectomy was still higher than that of lobectomy. No severe postoperative complications (grade III-grade V) occurred in either study group. Most of the postoperative complications were air leak, subcutaneous emphysema, and temporary arrhythmia (Supplementary table 2).

Regarding the image quality of the robotic endoscope holder and the human-assisted single-port surgery, no significant difference was found in the numbers of endostaples used (Table 3). However, the frequency of image location in the marginal zone was lower in the HA group than in the EA group. To investigate the effects of different procedures on the two groups, we divided the matched cohort into wedge and anatomic resection subgroups. In the wedge resection subgroup, the intercepted image quality revealed no significant difference, all of the intercepted image fall into central and intermediate zone $(P=0.40$ and 0.54 , respectively). In the anatomic resection subgroup, the frequency of endostaples in the marginal zone of intercepted surgical images significantly differed between HA and EA subgroups $(P<0.01)$.

\section{Discussion}

For various surgical interventions, single-port VATS has been approved as safe, and it produces noninferior treatment outcomes compared with conventional open and multiport VATS [9-11]. In the basic configuration, multiport VATS requires two or three participants depending on the complexity of surgery. In SPVATS, one surgeon and one experienced assistant were sufficient to complete all variations of the surgery. Sometimes, it is difficult for a surgeon and assistant to maintain a proper social distance during SPVATS. Based on the relevant research on COVID-19, maintaining a physical distance of $1 \mathrm{~m}$ or more can prevent the spread of COVID-19 infection [12]. Furthermore, the high prevalence of COVID19 among asymptomatic cases as well as the false-negative rate of molecular test results might cause the infection of health care workers [13-15]. Recently, Dr. Ayhan published an article about the perioperative infection rate of COVID19 in gynecologic cancer patients [16]. $6.7 \%$ of gynecologic cancer patients undergoing major surgery patients developed COVID-19 infection. Despite a relatively lower infection rate, minimizing the number of people involved in surgery and maintaining proper social distancing might help reduce and control cluster infections in hospitals.

With the introduction of the robotic arm, the staff requirements for surgery might become more flexible. In Taiwan and other countries, some concerns have been raised regarding the regulations of residents' work hours, and the decline in residency applications to surgical departments has caused staff shortages [17, 18], especially 
Table 1 Patient demographics and baseline characteristics before and after propensity matching (1:1)

\begin{tabular}{|c|c|c|c|c|c|c|}
\hline \multirow[t]{2}{*}{ Variable } & \multicolumn{3}{|l|}{ Entire cohort } & \multicolumn{3}{|c|}{ Propensity score matching } \\
\hline & $\begin{array}{l}\text { HA group } \\
(n=156)\end{array}$ & $\begin{array}{l}\text { EA group } \\
(n=57)\end{array}$ & $P$ value & $\begin{array}{l}\text { HA group } \\
(n=52)\end{array}$ & $\begin{array}{l}\text { EA group } \\
(n=52)\end{array}$ & $P$ value \\
\hline Age & $60.5 \pm 13.5$ & $60.3 \pm 15.6$ & 0.91 & $57.3 \pm 15.3$ & $59.9 \pm 16$ & 0.41 \\
\hline Gender & & & 0.70 & & & 0.84 \\
\hline Male & $84(53.8 \%)$ & $29(50.9 \%)$ & & $27(51.9 \%)$ & $26(50.0 \%)$ & \\
\hline Female & $72(46.2 \%)$ & $28(49.1 \%)$ & & $25(48.1 \%)$ & $26(50.0 \%)$ & \\
\hline Smoking history & & & 0.22 & & & 0.82 \\
\hline Yes & $58(37.2 \%)$ & $16(28.1 \%)$ & & $15(28.8 \%)$ & $14(26.9 \%)$ & \\
\hline No & $98(62.8 \%)$ & $41(71.9 \%)$ & & $37(71.2 \%)$ & $38(73.1 \%)$ & \\
\hline ACS history & & & 1.00 & & & 1.00 \\
\hline Yes & $8(5.1 \%)$ & $3(5.3 \%)$ & & $1(1.9 \%)$ & $2(3.8 \%)$ & \\
\hline No & $148(94.9 \%)$ & $54(94.8 \%)$ & & $51(98.1 \%)$ & $50(96.2 \%)$ & \\
\hline COPD & & & 0.73 & & & 0.62 \\
\hline Yes & $7(4.5 \%)$ & $3(5.3 \%)$ & & $1(1.9 \%)$ & $3(5.8 \%)$ & \\
\hline No & $149(95.5 \%)$ & $54(94.7 \%)$ & & $51(98.1 \%)$ & $49(94.2 \%)$ & \\
\hline Renal disease & & & 1.00 & & & \\
\hline Yes & $5(3.2 \%)$ & $1(1.8 \%)$ & & $2(3.8 \%)$ & $0(0 \%)$ & 0.50 \\
\hline No & $151(96.8 \%)$ & $56(98.2 \%)$ & & $50(96.2 \%)$ & $52(100 \%)$ & \\
\hline Body Mass Index & $24.7 \pm 4.3$ & $24.4 \pm 3.8$ & 0.68 & $24.1 \pm 4.4$ & $25 \pm 3.4$ & 0.26 \\
\hline ECOG & & & 0.002 & & & 1.00 \\
\hline 0 & $153(98.7 \%)$ & $50(87.7 \%)$ & & $50(96.2 \%)$ & $50(96.2 \%)$ & \\
\hline 1 & $2(1.3 \%)$ & $7(12.3 \%)$ & & $2(3.8 \%)$ & $2(3.8 \%)$ & \\
\hline Operation type & & & 0.30 & & & 0.42 \\
\hline Wedge & $51(32.7 \%)$ & $23(40.4 \%)$ & & $19(36.5 \%)$ & $23(44.2 \%)$ & \\
\hline Anatomic resection & $105(67.3 \%)$ & $34(59.6 \%)$ & & $33(63.5 \%)$ & $29(55.8 \%)$ & \\
\hline Diagnosis & & & 0.73 & & & 1.00 \\
\hline Malignancy & $114(73.1 \%)$ & $43(75.4 \%)$ & & $37(71.2 \%)$ & $38(73.1 \%)$ & \\
\hline Benign & $46(26.9 \%)$ & $14(24.6 \%)$ & & $15(28.8 \%)$ & $14(26.9 \%)$ & \\
\hline Lesion location & & & 0.49 & & & 0.24 \\
\hline RUL & $37(23.7 \%)$ & $14(24.6 \%)$ & & $12(23.1 \%)$ & $12(23.1 \%)$ & \\
\hline RML & $19(12.2 \%)$ & $4(7 \%)$ & & $5(9.6 \%)$ & $4(7.7 \%)$ & \\
\hline RLL & $36(23.1 \%)$ & $9(15.8 \%)$ & & $17(32.7 \%)$ & $8(15.4 \%)$ & \\
\hline LUL & $38(24.4 \%)$ & $17(29.8 \%)$ & & $10(19.2 \%)$ & $16(30.8 \%)$ & \\
\hline LLL & $26(16.7 \%)$ & $13(22.8 \%)$ & & $8(15.4 \%)$ & $12(23.1 \%)$ & \\
\hline Post OP complication & & & 0.66 & & & 1.00 \\
\hline Yes & $4(2.6 \%)$ & $2(3.5 \%)$ & & $1(1.9 \%)$ & $1(1.9 \%)$ & \\
\hline No & $152(97.4 \%)$ & $55(96.5 \%)$ & & $51(98.1 \%)$ & $51(98.1 \%)$ & \\
\hline
\end{tabular}

ACS history acute coronary syndrome history, COPD chronic obstructive pulmonary disease, ECOG Eastern Cooperative Oncology Group scale

during the COVID-19 pandemic. Taiwan's efforts to fight COVID-19 have been successful, and most residents have been able to maintain a normal social life $[19,20]$. As a dedicated medical center for frontline care of patients, our institute follows existing treatment guidelines [21], and situations of medical staff shortages are even simulated in advance. In this retrospective study, we attempted to replace a human assistant with a robotic arm to reduce the staff required during surgery, a factor that has emerged because of the COVID-19 pandemic. In our preliminary results, no significant difference was found in perioperative surgical results between the two groups. However, regarding staffing requirements in the EA group, $100 \%$ of wedge resections, $95 \%$ of segmentectomies, and $64 \%$ of lobectomies required only one surgeon. Additionally, no difference was measured in the postoperative complication rate. In our series, the robotic endoscope holder enabled surgeries to be performed with limited staff. Based on 
Table 2 Perioperative parameters of robotic endoscope holder and human assisted single port surgery

\begin{tabular}{|c|c|c|c|c|c|c|}
\hline \multicolumn{7}{|l|}{ Whole cohort } \\
\hline \multirow[t]{2}{*}{ Variable } & \multicolumn{3}{|c|}{ Unadjusted cohort } & \multicolumn{3}{|c|}{ Propensity score matching } \\
\hline & $\begin{array}{l}\text { HA group } \\
(n=156)\end{array}$ & $\begin{array}{l}\text { EA group } \\
(n=57)\end{array}$ & $P$ value & $\begin{array}{l}\text { HA group } \\
(n=52)\end{array}$ & $\begin{array}{l}\text { EA group } \\
(n=52)\end{array}$ & $P$ value \\
\hline Preparation time & $14.6 \pm 3.8$ & $14.6 \pm 2.8$ & 0.98 & $14.5 \pm 4.2$ & $14.7 \pm 2.9$ & 0.78 \\
\hline Operative time & $140 \pm 51.8$ & $136.4 \pm 57.6$ & 0.66 & $125.1 \pm 53.3$ & $135.8 \pm 59.8$ & 0.33 \\
\hline Blood loss & $25.6 \pm 8.1$ & $25.4 \pm 6.6$ & 0.91 & $25.2 \pm 9.4$ & $25.2 \pm 6.7$ & 1.00 \\
\hline Chest tube duration (h) & $60.1 \pm 34.5$ & $59.4 \pm 33.6$ & 0.90 & $53.7 \pm 25.1$ & $58 \pm 34.4$ & 0.47 \\
\hline Post OP stay (h) & $77.9 \pm 35.3$ & $79.2 \pm 33.1$ & 0.82 & $70.7 \pm 24$ & $80.4 \pm 33.9$ & 0.10 \\
\hline Lesion size $(\mathrm{cm})$ & $1.94 \pm 1.31$ & $1.63 \pm 1.52$ & 0.16 & $1.56 \pm 0.95$ & $1.54 \pm 1.49$ & 0.91 \\
\hline PreOP triflow & $2.61 \pm 0.69$ & $2.72 \pm 0.54$ & 0.45 & $2.61 \pm 0.58$ & $2.68 \pm 0.55$ & 0.54 \\
\hline Day 1 triflow & $1.53 \pm 0.92$ & $1.75 \pm 0.79$ & 0.11 & $1.62 \pm 0.90$ & $1.77 \pm 0.76$ & 0.37 \\
\hline Day 2 triflow & $2.05 \pm 0.81$ & $2.39 \pm 0.73$ & 0.07 & $2.12 \pm 0.71$ & $2.38 \pm 0.72$ & 0.06 \\
\hline Day 3 triflow & $2.45 \pm 0.70$ & $2.55 \pm 0.63$ & 0.33 & $2.48 \pm 0.61$ & $2.65 \pm 0.59$ & 0.17 \\
\hline Post OP complication & & & 0.66 & & & 1.000 \\
\hline Yes & $4(2.6 \%)$ & $2(3.5 \%)$ & & $1(1.9 \%)$ & $1(1.9 \%)$ & \\
\hline No & $152(97.4 \%)$ & $55(96.5 \%)$ & & $51(98.1 \%)$ & $51(98.1 \%)$ & \\
\hline \multicolumn{7}{|l|}{ Surgeondemand } \\
\hline \multicolumn{7}{|l|}{ Wedge } \\
\hline 1 & $0(0 \%)$ & $23(100 \%)$ & $<0.01$ & $0(0 \%)$ & $23(100 \%)$ & $<0.01$ \\
\hline 2 & $51(100 \%)$ & $0(0 \%)$ & & $19(100 \%)$ & $0(0 \%)$ & \\
\hline \multicolumn{7}{|l|}{ Segmentectomy } \\
\hline 1 & $0(0 \%)$ & $19(95 \%)$ & $<0.01$ & $0(0 \%)$ & $16(94.1 \%)$ & $<0.01$ \\
\hline 2 & $44(100 \%)$ & $1(5 \%)$ & & $21(100 \%)$ & $1(5.9 \%)$ & \\
\hline \multicolumn{7}{|l|}{ Lobectomy } \\
\hline 1 & $0(0 \%)$ & $9(64.3 \%)$ & $<0.01$ & $0(0 \%)$ & $7(58.3 \%)$ & $<0.01$ \\
\hline 2 & $61(100 \%)$ & $5(35.7 \%)$ & & $12(100 \%)$ & $5(41.7 \%)$ & \\
\hline
\end{tabular}

Table 3 Image quality comparison of ENDOFIX ${ }^{\text {exo }}$ and human-assisted single-port surgery after matching

\begin{tabular}{|c|c|c|c|c|}
\hline & HA group & EA group & Total & $P$ value \\
\hline \multicolumn{5}{|c|}{ Anatomic resection $(n=62)$} \\
\hline No of endostaple & $8.9 \pm 3.4$ & $9.8 \pm 2.9$ & $9.4 \pm 3.2$ & 0.28 \\
\hline \multicolumn{5}{|l|}{ Image quality } \\
\hline Central & $7 \pm 2.1$ & $6.2 \pm 2.6$ & $6.7 \pm 2.3$ & 0.19 \\
\hline Intermediate & $1.8 \pm 1.5$ & $2.6 \pm 1.6$ & $2.2 \pm 1.6$ & 0.06 \\
\hline Marginal & $0.1 \pm 0.2$ & $1 \pm 0.6$ & $0.5 \pm 0.6$ & $<0.01$ \\
\hline \multicolumn{5}{|l|}{ Wedge $(n=42)$} \\
\hline No of endostaple & $4.7 \pm 1.7$ & $4.7 \pm 2.1$ & $4.7 \pm 1.9$ & 0.93 \\
\hline \multicolumn{5}{|l|}{ Image quality } \\
\hline Central & $4.2 \pm 1.6$ & $4.7 \pm 2$ & $4.4 \pm 1.8$ & 0.40 \\
\hline Intermediate & $0.2 \pm 0.9$ & $0.1 \pm 0.3$ & $0.1 \pm 0.6$ & 0.54 \\
\hline Marginal & $0 \pm 0$ & $0 \pm 0$ & $0 \pm 0$ & - \\
\hline \multicolumn{5}{|c|}{ Whole group $(n=104)$} \\
\hline No of endostaple & $7.4 \pm 3.5$ & $7.6 \pm 3.6$ & $7.5 \pm 3.6$ & 0.79 \\
\hline \multicolumn{5}{|l|}{ Image quality } \\
\hline Central & $6 \pm 2.4$ & $5.5 \pm 2.5$ & $5.8 \pm 2.4$ & 0.35 \\
\hline Intermediate & $1.3 \pm 1.5$ & $1.5 \pm 1.7$ & $1.4 \pm 1.6$ & 0.47 \\
\hline Marginal & $0 \pm 0.2$ & $0.5 \pm 0.7$ & $0.3 \pm 0.6$ & $<0.01$ \\
\hline
\end{tabular}


Table 4 Manpower costs analysis of unisurgeon single port VATS in wedge, segmentectomy and lobectomy

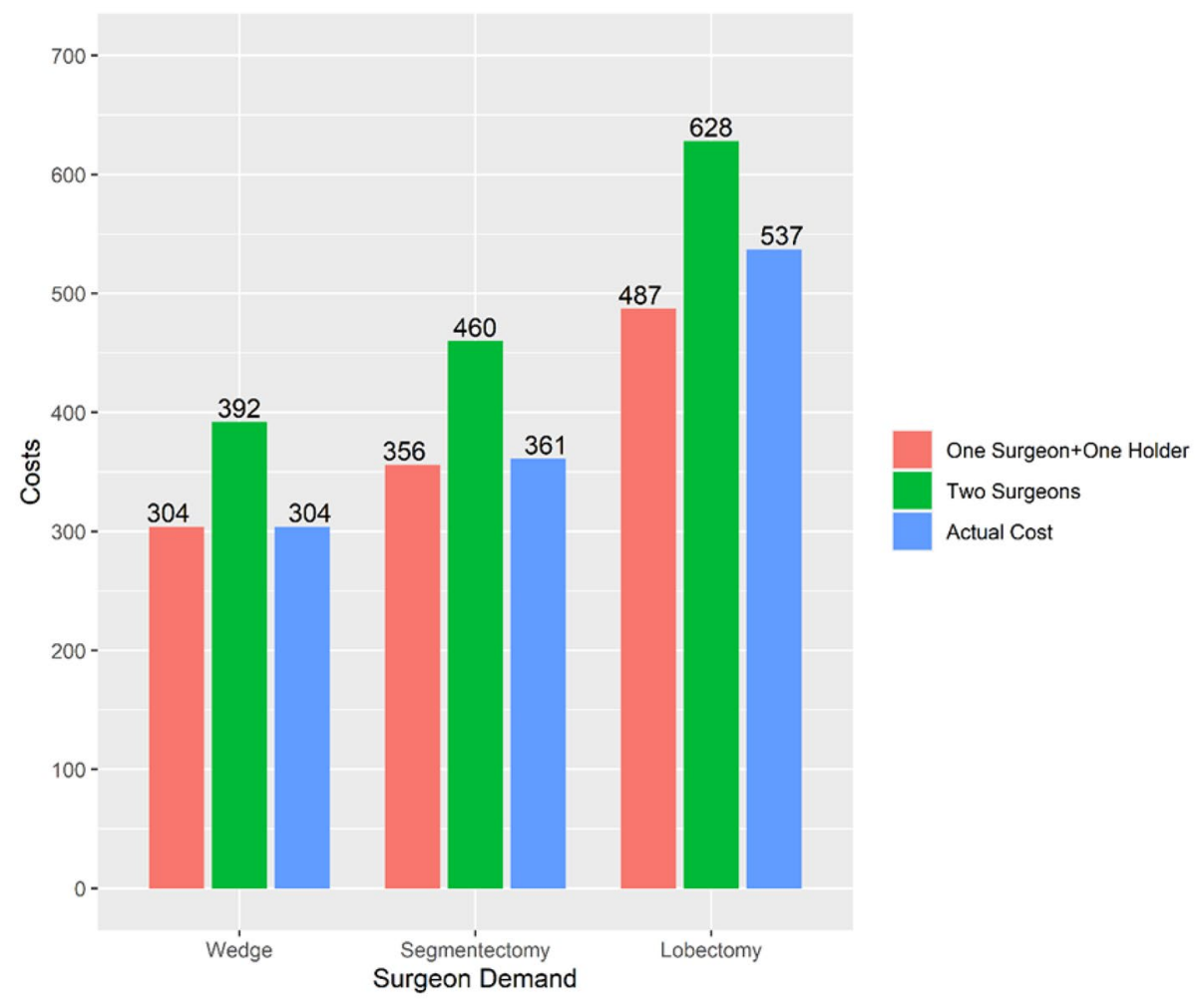

\begin{tabular}{|c|c|c|c|c|c|c|}
\hline & $\begin{array}{l}\text { Average OP* time } \\
(\min )\end{array}$ & Two surgeons & Surgeon + Holder & Successful rate & Actual cost & $\begin{array}{l}\text { Reduced } \\
\text { propor- } \\
\text { tion }\end{array}$ \\
\hline Wedge & 125.0 & 392 & 304 & $100 \%$ & 304 & $23 \%$ \\
\hline Secmentectomy & 146.5 & 460 & 356 & $95 \%$ & 361.2 & $22 \%$ \\
\hline Lobectomy & 200.2 & 628 & 487 & $64 \%$ & 537 & $15 \%$ \\
\hline
\end{tabular}

OP*: whole operation time

Cost/min of surgeon: 1.57 USD cost/min of Robotic Holder: 0.86 USD

the medical quality and cost considerations, the robotic endoscope holder might replace the role of the assistant in uniport VATS surgery. Manpower costs can be reduced by $23 \%$ in wedge resection, then, in order, $22 \%$ and $15 \%$ of labor costs reduced in segmentectomy and lobectomy. The detailed calculation results were listed in (Table 4). This improvement plan may help to overcome the workforce shortage and effectively reduce costs in health care; further prospective studies are required.

The robotic scope holder system provides a stable surgical field, even in extreme endoscope positions [22], and negates the need for an assistant; these are considerable advantages over manual camera control by a human assistant. However, high surgical image quality requires not only stability but also the target area to be placed in the center of the endoscope image. In the present retrospective study, we devised image zones to evaluate the degree of achievement in robotic endoscope holder usage. The HA group played a favorable role in preventing the target lesion location from being in the marginal zone of the endoscope image during anatomic resection. In most images, the endoscope stapler passed through blood vessels or lung parenchyma in the central or central-intermediate zone in the HA group. In the EA group, stapler passing in the marginal zone of images was relatively frequent. This may have been because the human assistant could actively adjust the scope to most appropriate lens angle and direction. Even if the robotic endoscope holder is designed to be similar to a bionic arm, it has a thicker scope holder and a more limited joint mobility than a human being has. This can cause collision and interference 
between the endostaple and robotic arms (Video 1). This is also the main reason for the failure of six unisurgeon single port VATS surgeries.

Pleural symphysis also poses a challenge for minimally invasive surgery. In series reports of incidents of unexpected conversion to thoracotomy during VATS, vascular injury and pleural adhesion were the primary causes [23]. Dense mediastinal side pleural adhesion also increased the chances of unexpected vascular injury during operation, as we noted in our previous report [24]. With the help of robotic endoscope holders, surgeons may face surgical challenges such as dense adhesion more cautiously and without the hurdle of unstable vision. Even when we encountered a patient who had undergone previous surgery, we nevertheless started the surgery with the same wound and slowly detached the adhesion lung tissue from the wound. Fortunately, we did not encounter a major pulmonary vessel injury in this cohort. However, we had anticipated such a crisis, and thus, we opted to let the most experienced surgeon try unisurgeon SPVATS. If we encounter a bleeding accident during surgery in the future, we will deal with it in accordance with the standard procedure for dealing with intraoperative bleeding. After all, patient safety comes before displays of bravura.

Some limitations of the current study merit mention. First, although propensity matching may reduce the bias inherent in a comparison of two surgical equipment configurations, prospective randomized trials are needed to confirm our findings. Second, this study was a pilot study investigating the feasibility of robotic endoscope holder assisted SPVATS with limited staff. During our study, we had to convert to a different stapler because the previously used one was out of stock temporarily due to COVID-19. In some scenario, the use of a thinner shaft stapler with a narrow placement tip and an anvil dramatically reduced the time spent on passing though the target vessels compared with use of the previous straight placement stapler; it also solved some of the problems of collision between the stapler and robotic arm (Video 1). Third, whether a single-surgeon single-port VATS is more feasible to perform in segmentectomy than in lobectomy still needs more verification. Most segmentectomy performed in the EA and HA groups were either a combined segmentectomy or single segmentectomy of the upper and middle lobe. The complex segmentectomy of the lower lobe or subsegmentectomy will be the focus of our next work. A more complex intersegment plane would be a major challenge for a unisurgeon SPVATS. Finally, this was only a single center, single surgical team experience of performing unisurgeon single-port VATS surgery.

Some limitations of the current study merit mention. First, although propensity matching may reduce the bias inherent in a comparison of two surgical equipment configurations, prospective randomized trials are needed to confirm our findings. Second, this study was a pilot study investigating the feasibility of robotic endoscope holder assisted SPVATS with limited staff. During our study, we had to convert to a different stapler because the previously used one was out of stock temporarily due to COVID-19. In some scenario, the use of a thinner shaft stapler with a narrow placement tip and an anvil dramatically reduced the time spent on passing though the target vessels compared with use of the previous straight placement stapler; it also solved some of the problems of collision between the stapler and robotic arm (Video 1). Third, whether a single-surgeon single-port VATS is more feasible to perform in segmentectomy than in lobectomy still needs more verification. Most segmentectomy performed in the EA and HA groups were either a combined segmentectomy or single segmentectomy of the upper and middle lobe. The complex segmentectomy of the lower lobe or subsegmentectomy will be the focus of our next work. A more complex intersegment plane would be a major challenge for a unisurgeon SPVATS. Finally, this was only a single center, single surgical team experience of performing unisurgeon single-port VATS surgery. The learning curve was an important issue that we need to address further. For the duration of the operation and any post-operative complications, there were no significant differences in our preliminary report. However, three methods of surgery are involved, so it may be difficult to be objective. Our expectation is that more surgeons and clinical applications will be added in the future, providing a more credible answer as more experience accumulates.

\section{Conclusion}

In conclusion, our preliminary results revealed that when performed by an experienced uniport surgeon, wedge resection can be performed without a human assistant (video 2). However, this held true for $95 \%$ of segmentectomy procedures (video 3 ) but only $64 \%$ of lobectomy procedures. Wedge resection was the most suitable procedure for unisurgeon single-port video-assisted thoracoscopic surgery without increasing setup time, operative time, or short-term complications. Verification of the technique's applicability for use in anatomic resections requires further investigation.

Supplementary Information The online version contains supplementary material available at https://doi.org/10.1007/s00464-022-09054-8.

Author contributions Study conception and design: CFW, MJH, CYW, DG-R, YKC. Acquisition of data: CFW, CYW, MJH, YKC. Analysis and interpretation of data: CFW, YBP, LYY. Drafting of manuscript: CFW, MJH, CYW. Critical revision: CFW, DG-R, MJH.

Funding The study is funded by Chang Gung Memorial Hospital Research Program: CMRPG5H0032 and CDRPG3J0031. Ching Feng 
Wu MD and Ming Ju Hsieh MD received the research funding programs. The authors acknowledge the statistical assistance provided by the Clinical Trial Center, Chang Gung Memorial Hospital, Linkou, Taiwan, which was founded by the Ministry of Health and Welfare of Taiwan; MOHW107-TDU-B-212-123005. Ching Yang Wu MD, Yin Kai, Chao MD, PHD, Diego Gonzalez-Rivas MD, received no financial support for the research, authorship, and/or publication of this article.

\section{Declarations}

Disclosures Ching Feng Wu MD, Ching Yang Wu MD, Yin Kai, Chao MD, PHD, Diego Gonzalez-Rivas MD, Ming Ju Hsieh MD, Yu Bin Pan MS, Lan Yan Yang PHD declare no potential conflicts of interest with respect to the research, authorship, and/or publication of this article.

\section{References}

1. Roviaro G, Rebuffat C, Varoli F, Vergani C, Mariani C, Maciocco M (1992) Videoendoscopic pulmonary lobectomy for cancer. Surg Laparosc Endosc 2:244-247

2. Yan TD, Black D, Bannon PG, McCaughan BC (2009) Systematic review and meta-analysis of randomized and nonrandomized trials on safety and efficacy of video-assisted thoracic surgery lobectomy for early-stage non-small-cell lung cancer. J Clin Oncol 27(15):2553-2562

3. Bendixen M, Jørgensen OD, Kronborg C, Andersen C, Licht PB (2016) Postoperative pain and quality of life after lobectomy via video-assisted thoracoscopic surgery or anterolateral thoracotomy for early stage lung cancer: a randomised controlled trial. Lancet Oncol 17(6):836-844

4. Berfield KS, Farjah F, Mulligan MS (2019) Video-assisted thoracoscopic lobectomy for lung cancer. Ann Thorac Surg 107(2):603-609

5. Scott WJ, Allen MS, Darling G, Meyers B, Decker PA, Putnam JB et al (2010) Video-assisted thoracic surgery versus open lobectomy for lung cancer: a secondary analysis of data from the American College of Surgeons Oncology Group Z0030 randomized clinical trial. J Thorac Cardiovasc Surg 139(4):976-981; discussion 981-983.

6. Okada S, Tanaba Y, Sugawara H, Yamauchi H, Ishimori S, Satoh S (2000) Thoracoscopic major lung resection for primary lung cancer by a single surgeon with a voice-controlled robot and an instrument retraction system. J Thorac Cardiovasc Surg 120:414-415

7. Kunisaki C, Hatori S, Imada T, Akiyama H, Ono H, Otsuka Y et al (2004) Video-assisted thoracoscopic esophagectomy with a voice-controlled robot: the AESOP system. Surg Laparosc Endosc Percutan Tech 14(6):323-327

8. Gonzalez-Rivas D (2017) Unisurgeon' uniportal video-assisted thoracoscopic surgery lobectomy. J Vis Surg 3:163

9. Migliore M (2003) Efficacy and safety of single-trocar technique for minimally invasive surgery of the chest in the treatment of noncomplex pleural disease. J Thorac Cardiovasc Surg 126(5):1618-1623

10. Wu CF, Fernandez R, de la Torre M, Delgado M, Fieira E, Wu $\mathrm{CY}$ et al (2018) Mid-term survival outcome of single-port videoassisted thoracoscopic anatomical lung resection: a two-centre experience. Eur J Cardiothorac Surg 54(2):252-259
11. Bourdages-Pageau E, Vieira A, Lacasse Y, Figueroa PU (2020) Outcomes of uniportal vs multiportal video-assisted thoracoscopic lobectomy. Semin Thorac Cardiovasc Surg 32(1):145-151

12. Chu DK, Akl EA, Duda S, Solo K, Yaacoub S, Schünemann HJ, COVID-19 Systematic Urgent Review Group Effort (SURGE) study authors (2020) Physical distancing, face masks, and eye protection to prevent person-to-person transmission of SARSCoV-2 and COVID-19: a systematic review and meta-analysis. Lancet 395(10242):1973-1987

13. Böger B, Fachi MM, Vilhena RO, Cobre AF, Tonin FS, Pontarolo R (2021) Systematic review with meta-analysis of the accuracy of diagnostic tests for COVID-19. Am J Infect Control 49(1):21-29

14. Vandenberg O, Martiny D, Rochas O, van Belkum A, Kozlakidis Z (2021) Considerations for diagnostic COVID-19 tests. Nat Rev Microbiol 19(3):171-183

15. Kronbichler A, Kresse D, Yoon S, Lee KH, Effenberger M, Shin JI (2020) Asymptomatic patients as a source of COVID-19 infections: a systematic review and meta-analysis. Int J Infect Dis 98:180-186

16. Ayhan A, Oz M, Topfedaisi Ozkan N, Aslan K, Altintas MI, Akilli $\mathrm{H}$ et al (2021) Perioperative SARS-CoV-2 infection among women undergoing major gynecologic cancer surgery in the COVID-19 era: a nationwide, cohort study from Turkey. Gynecol Oncol 160(2):499-505

17. Lockley SW, Cronin JW, Evans EE, Cade BE, Lee CJ, Landrigan CP et al (2004) Effect of reducing interns' weekly work hours on sleep and attentional failures. N Engl J Med 351(18):1829-1837

18. Jagannathan J, Vates GE, Pouratian N, Sheehan JP, Patrie J, Grady MS, Jane JA (2009) Impact of the Accreditation Council for Graduate Medical Education work-hour regulations on neurosurgical resident education and productivity. J Neurosurg 110(5):820-827

19. Steinbrook R (2020) Contact tracing, testing, and control of COVID-19-learning from Taiwan. JAMA Intern Med 180(9):1163-1164

20. Seak CJ, Liu YT, Ng CJ, SPOT investigators (2020) Rapid responses in the emergency department of Linkou Chang Gung Memorial Hospital, Taiwan effectively prevent spread of COVID19 among healthcare workers of emergency department during outbreak: lessons learnt from SARS. Biomed J 43(4):388-391

21. Thoracic Surgery Outcomes Research Network, Inc, Antonoff M, Backhus L, Boffa DJ, Broderick SR, Brown LM, et al (2020) COVID-19 guidance for triage of operations for thoracic malignancies: a consensus statement from Thoracic Surgery Outcomes Research Network. J Thorac Cardiovasc Surg 160(2):601-605

22. Ohmura Y, Suzuki H, Kotani K, Teramoto A (2019) Comparative effectiveness of human scope assistant versus robotic scope holder in laparoscopic resection for colorectal cancer. Surg Endosc 33(7):2206-2216

23. Fourdrain A, De Dominicis F, Iquille J, Lafitte S, Merlusca G, Witte-Pfister A et al (2019) Intraoperative conversion during video-assisted thoracoscopy does not constitute a treatment failure. Eur J Cardiothorac Surg 55(4):660-665

24. Wu CF, de la Mercedes T, Fernandez R, Delgado M, Fieira E, Wu $\mathrm{CY}$ et al (2019) Management of intra-operative major bleeding during single-port video-assisted thoracoscopic anatomic resection: two-center experience. Surg Endosc 33(6):1880-1889

Publisher's Note Springer Nature remains neutral with regard to jurisdictional claims in published maps and institutional affiliations. 